\title{
Bisquinolinium compounds induce quadruplex- specific transcriptome changes in HeLa S3 cell lines
}

\author{
Rashi Halder', Jean-Francois Riou², Marie-Paule Teulade-Fichou ${ }^{3}$, Tancred Frickey ${ }^{4}$ and Jörg S Hartig ${ }^{1 *}$
}

\begin{abstract}
Background: Guanosine rich sequences capable of forming G-quadruplex (G4) motifs are enriched near the gene transcription start site (TSS) in the human genome. When probed at the single gene level, G-quadruplex motifs residing in promoter regions show substantial effects on gene transcription. Moreover, further changes in transcription levels are noticed when G4-motifs are targeted with G-quadruplex-specific small molecules.

Results: Global studies concerning general changes of the transcriptome via targeting promoter-based Gquadruplex motifs are very limited and have so far only been carried out with compounds displaying weak selectivity for quadruplex sequences. Here we utilize two G-quadruplex-specific bisquinolinium derivatives PhenDC3 and 360A and investigate their effects on the expression of the HeLa S3 transcriptome. Our results show widespread changes in the transcriptome with specificity for genes with G-quadruplex motifs near their transcription start sites (TSS). Using real-time PCR we further confirmed the specificity of PhenDC3 and 360A as potent molecules to target G-quadruplex-regulated genes.
\end{abstract}

Conclusions: Specific effects on quadruplex-containing genes have been observed utilizing whole-transcriptome analysis upon treatment of cultured cells with quadruplex-selective bisquinolinium compounds.

\section{Background}

G-quadruplex motifs are four-stranded DNA conformations adopted by certain guanosine-rich sequences [1] abundant in the human genome [2,3]. Interestingly, these G-quadruplex motif-forming sequences show a pronounced positional bias and consequently a wide range of biological functions have been predicted for Gquadruplex motifs, including roles in transcription [4-6], translation $[7,8]$, replication [9], nucleosome positioning [10,11], CpG methylation [12], recombination [13] and splicing [14]. Due to their high over-representation near transcription start sites (TSS) [15], the role of G-quadruplex motifs as transcriptional regulators has been studied most intensively (reviewed in [16-19]). The first experimentally verified biological function for a G-quadruplex motif was the transcriptional regulation of the $c$ -

\footnotetext{
* Correspondence: joerg.hartig@uni-konstanz.de

'Department of Chemistry and Konstanz Research School Chemical Biology (KoRS-CB), University of Konstanz, Universitätsstraße 10, 78457 Konstanz, Germany

Full list of author information is available at the end of the article
}

$M Y C$ gene [20,21]. Similar modulatory effects in transcription have since been demonstrated for quadruplex motifs in the promoter regions of $c-M Y B$ [22], VEGF [23], $c-K I T$ [24], KRAS [25], HRAS [26], PDGFR- $\beta$ [27] and $B C L-2$ [28] expression. In several studies a small molecule specific to G-quadruplex motifs was used to demonstrate that quadruplex formation in vivo results in a perturbation of transcription (reviewed in $[19,29,30])$. However, since more than $55 \%$ of the genes in the human genome harbor at least one potential Gquadruplex motif $1 \mathrm{~kb}$ up- or downstream of the TSS [5], it is of interest to investigate whether addition of quadruplex-interacting compounds affects transcription of many or most of these genes.

Using the G-quadruplex-stabilizing molecule TMPyP4 [31], Verma et. al. [5] and Mikami-Terao et. al. [32] showed genome-wide effects on transcription in HeLa S3 and K562 cells, respectively. However, the specificity of the observed effects can be questioned because TMPyP4 is reported to show only poor selectivity for G-quadruplex motifs compared to e.g. duplex DNA [33]. The two studies
C Biomed Central

() 2012 Hartig et al; licensee BioMed Central Ltd. This is an open access article distributed under the terms of the Creative Commons Attribution License (http://creativecommons.org/licenses/by/2.0), which permits unrestricted use, distribution, and reproduction in any medium, provided the original work is properly cited. 
identified a rather small set of affected genes at prolonged (48 h) treatment using high $(100 \mu \mathrm{M})$ concentrations of TMPyP4. In these studies, only 69 [5] and 87 [32] perturbations of mRNA levels were observed. Moreover, porphyrin molecules were reported to have cytotoxic effects starting at $50 \mu \mathrm{M}[34,35]$ and are photoactive [36,37], potentially resulting in harmful side-effects. More recently, using a single-chain antibody specific to G-quadruplex motifs, Fernando et. al. [38] determined 1,767 significant differentially expressed genes in a HGC-27 cell line. It should be noted that the choice of the cell line has very likely a strong impact on the outcome of whole-transcriptome examinations as described here. For example, a direct comparison of differentially expressed genes upon compound incubation between two cell lines in our opinion makes only sense if the compared genes are indeed expressed in both cell lines. Hence the immediate comparison of transcripome datasets between different cell lines will result in many apparent contradictions that might result from different expression states of the genes in different cell lines. In a further study, G-quadruplex motifs were selectively pulled down from HT1080 cells using a biotinylated bisquinolinium derivative [39]. However, in the latter study, an assignment of preferential genomic location for G4-motifs was not achieved, thereby limiting the insights gained regarding the in vivo-function of Gquadruplex formation. In conclusion, further genome-wide studies of transcriptional changes mediated by quadruplexspecific compounds are needed in order to judge the extent to which G-quadruplexes influence transcription.

In the present work we investigate genome-wide effects on transcription in a HeLa S3 cell line via stabilizing Gquadruplex motifs using the quadruplex-selective bisquinolinium compounds PhenDC3 and 360A (Figure 1A-B). Both molecules derived from the triazine derivative 12459 [40] and have been extensively studied for their Gquadruplex specificity both in vitro [41-43] and in vivo [44-47]. In addition we used the bisquinolinium compound $8979 \mathrm{~A}$ as a control as this compound also contains two quinolinium residues (see Figure $1 \mathrm{C}$ ) but is known for its poor affinity and selectivity for DNA Gquadruplex motifs [43]. Of the 24,651 genes probed in this work by a transcriptome array, 2,212 and 526 genes significantly changed their expression when treated with PhenDC3 or 360A, respectively. Of these, $81.5 \%$ and $70 \%$ had at least one G-quadruplex motif near their TSS. We confirmed the specificity of the target G-quadruplexes by real-time PCR for 8 individual genes.

\section{Results and discussion}

Transcriptome profiling of HeLa S3 cells treated with PhenDC3 or 360A

Whole genome expression of genes was determined using Illumina HumanHT-12v4 expression BeadChips

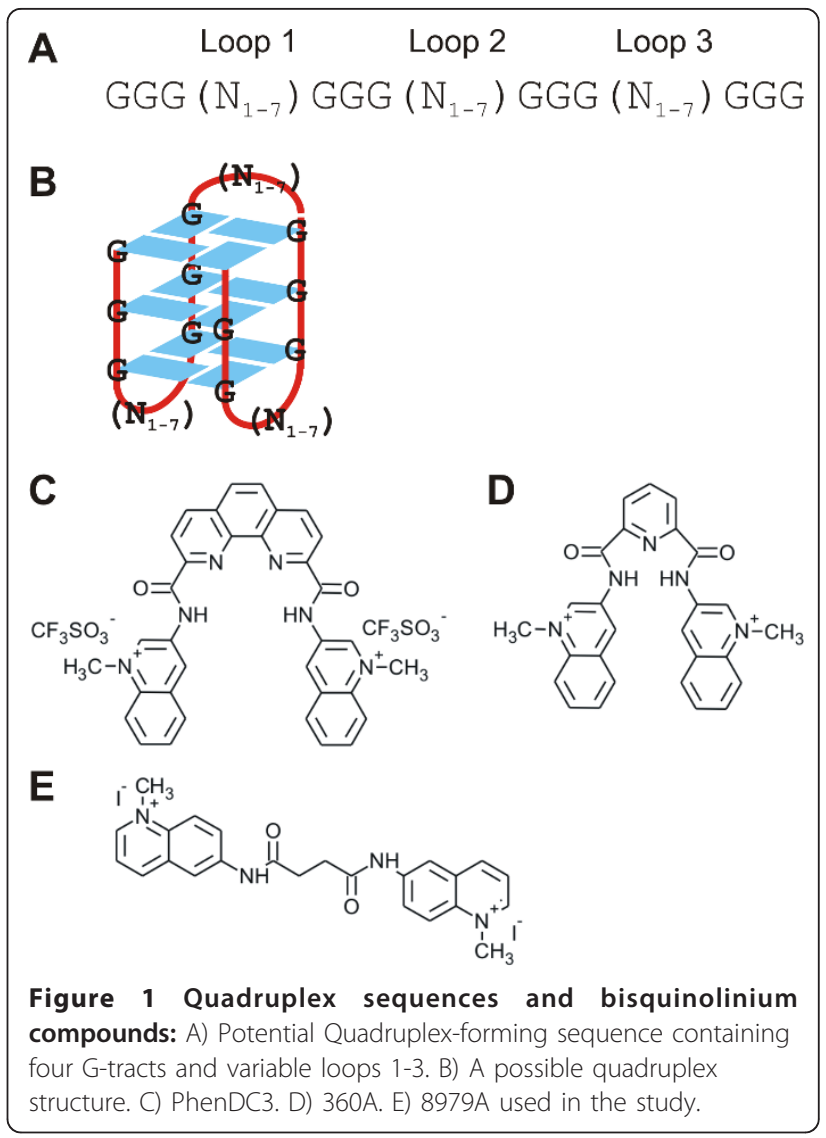

after 48 hours of treatment with $10 \mu \mathrm{M}$ of the G-quadruplex-specific molecules PhenDC3 or 360A in HeLa S3 cells. To account for non-specific effects mediated by the bisquinolinium compounds, 8979A (Figure 1C) was used as a control as it has a similar structure and charges but displays a very limited binding affinity to Gquadruplex motifs [43]. All molecule treatments were performed in triplicate. For analysis, the intensity values of each sample were subjected to 'quantile normalization'. The expression data was analyzed using the CLANS software [48]. First, the two control sets (untreated and 8979A-treated) samples were compared to confirm the non-specificity of the control molecule. Our analysis identified only 85 and 117 illumina identifiers down- and up-regulated, respectively, under the control condition. Next, we compared the expression of PhenDC3- or 360A-treated samples with the control samples (untreated and 8979A treated) to exclude those genes which might have been affected in their expression by treatment with the control molecule. We therefore considered the untreated and the 8979A-treated sample each as a control. Only genes showing a correlation coefficient of $\geq 0.9$ to our conditions of interest (Figure 2) and an anova P-value $\leq 0.05$ were taken into account and constitute our set of differentially expressed 


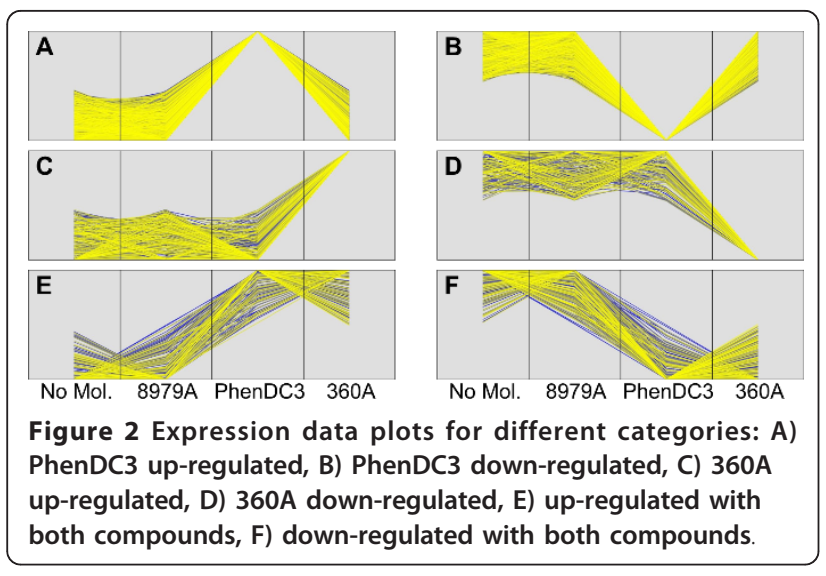

genes. The detailed gene list is given in Additional file 2: Table S4.

\section{G-quadruplex motifs in differentially expressed genes}

Using the Genbank files from NCBI (build 36.2), we could map 52,980 G-quadruplex motifs within the $2 \mathrm{~kb}$ region up- and downstream of the TSS of 17,384 mRNAs (see methods for details). For further analysis we considered only those mRNAs for which the Illumina IDs are present in the differentially expressed set, which gave a final set of 1,157 down- and 1,529 up-regulated Illumina IDs for PhenDC3 and 249 down- and 401 up-regulated for 360A, respectively (Table 1 ). We found that, in terms of differentially expressed genes, $81.5 \%(1,804$ out of 2,212$)$ of PhenDC3-treated and 70\% (369 out of 526) of 360A-treated genes have at least one PG4 motif within the $\pm 2 \mathrm{~kb}$ region of the TSS, which is significantly higher (hypergeometric test; $7.13 \mathrm{E}^{-98}$ (PhenDC3) and $0.01(360 \mathrm{~A})$ ) than the whole genome average $(67.3 \% ; 16,607$ out of 24,651$)$. This indicated a specificity and noticeable effect on transcription of both bisquinolinium compounds towards G-quadruplex motifs under our experimental conditions. We also found that 185 and 287 genes were up- or down-regulated in both bisquinolinium-treated samples, respectively (Figure $2 \mathrm{E}-\mathrm{F})$.
Next we analyzed whether we could detect any difference in the distribution of G-quadruplex motifs in our up- and down-regulated set of genes. Surprisingly, the normalized distribution of down-regulated mRNAs shows a higher occurrence of one or two G-quadruplex motifs in their core promoter region $( \pm 2 \mathrm{~kb}$ of TSS) compared to unchanged or up-regulated mRNAs. In contrast to this, genes with four or more G-quadruplexes are over-represented in the set of up-regulated mRNAs (Figure 3). To further validate the microarray results, we randomly chose 8 genes for quantitative real time PCR: Four genes showed changes in expression under treatment with both molecules (CTPS2, DPY30, $D I O 2, E G F R)$ and 2 genes each showed changes with either one of the molecules (PhenDC3: CANX and AK1; 360A: PFKM and SH3GL1). To further check whether the changes in gene expression show a dependency with respect to compound concentrations the cells were treated with 4 different concentrations (10,20, 50 and 100 $\mu \mathrm{M})$ of the G-quadruplex specific molecules PhenDC3 and 360A for 48 hours. We also used minimum (10 $\mu \mathrm{M})$ and maximum $(100 \mu \mathrm{M})$ concentrations of the control bisquinolinium 8979A. In addition, expression of TGOLN2 was also analyzed as a control because it did not appear to induce significant changes in expression in the microarray experiments. As a result of the realtime PCR data we were able to ascertain that all genes exhibited changes in their mRNA levels with a trend consistent to the microarray results (see Table 2). Except $C A N X$, all genes showed a concentration-dependent increase or decrease in expression.

\section{Effect of position and strand occupancy of G-quadruplex motifs}

To investigate the effect of the position of G-quadruplexes with respect to the TSS and strand orientation, we further compared the number of G-quadruplexes in the up- and down-regulated genes with the set of genes showing no significant change in expression that also harbored quadruplexes in their $\pm 2 \mathrm{~kb}$ region (non-

Table 1 The distribution of G4 motifs in differentially expressed genes (Illumina IDs) after PhenDC3 and 360A treatment

\begin{tabular}{|c|c|c|c|c|}
\hline & Illumina IDs & No. of Genes (with G4 motif) & No. of G4 motifs & Average loop length \\
\hline unchanged $^{a}$ & & & 9,456 & 3.7 \\
\hline 8979A -up-regulated ${ }^{b}$ & 117 & 83 & & \\
\hline 8979A -down-regulated ${ }^{b}$ & 85 & 55 & & \\
\hline PhenDC3 - up-regulated ${ }^{b}$ & 1529 & $1242(975)$ & 3,566 & 3.7 \\
\hline PhenDC3 - down-regulated ${ }^{b}$ & 1157 & $970(829)$ & 2,405 & 3.7 \\
\hline $360 A$ - up-regulated ${ }^{b}$ & 401 & $312(233)$ & 772 & 3.8 \\
\hline $360 A$ - down-regulated ${ }^{b}$ & 249 & $215(136)$ & 337 & 3.8 \\
\hline
\end{tabular}

non-differentially expressed genes common in all the gene sets, anova $>0.75$

${ }^{b}$ differentially expressed genes, anova $\leq 0.5$ and correlation $\geq 0.9$ 


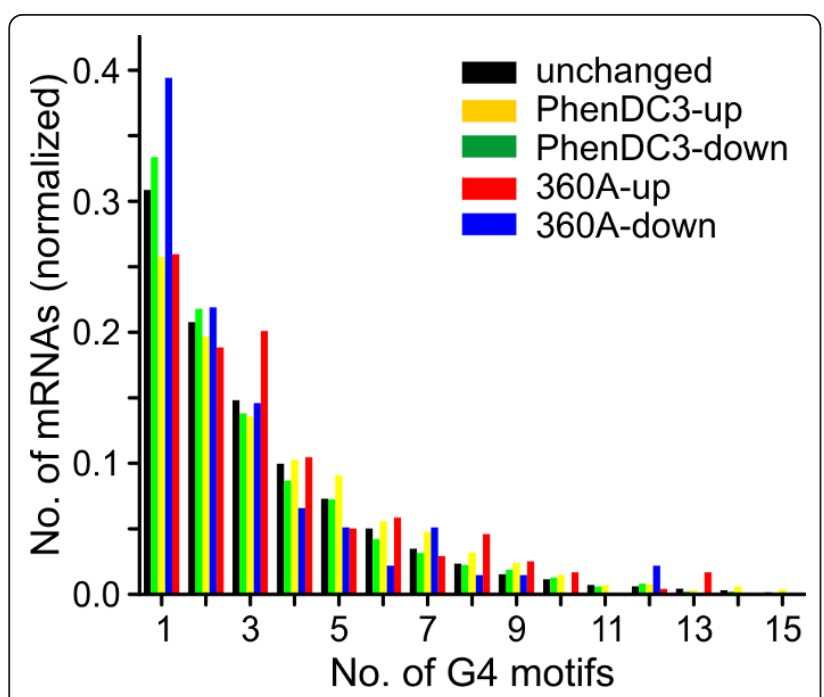

Figure 3 Distribution of the number of G-quadruplex motifs in the core promoter region of differentially expressed and unchanged genes. Data with more than $16 \mathrm{G}$-quadruplex motifs in the promoter are not shown (less than $1 \%$ of total)

differentially expressed genes common between all the gene sets, anova $>0.75$ ). These were divided into 4 subsets each: 1) af: G4-motifs present after TSS on forward strand, 2) ar: after TSS on reverse strand, 3) bf: before TSS on forward strand and 4) br: before TSS on reverse strand (Additional file 1: Table S1). Interestingly, we found that the number of G-quadruplexes in category af and br are significantly higher for the up- and down- regulated genes (both examined molecules) than unchanged/non-differentially expressed genes and have lower representation for category bf (hypergeometric test, $\mathrm{p}<0.05$, Figure 4).

\section{G-quadruplex motif loop length and composition in differentially expressed genes}

In order to search for a consensus motif or common feature that might be responsible for the differing behavior in non-affected and differentially expressed genes in response to the compound treatment, we investigated the difference in sequence composition between the Gquadruplex motifs present in differentially expressed genes versus genes with unchanged expression. The loop length and its nucleotide composition has been extensively studied earlier and both are known to affect the thermodynamic stability of G-quadruplex motifs [49-51]. Hence, we first examined the average loop length of G-quadruplex motifs occurring in differentially expressed versus non-differentially expressed genes. As shown in Table 1 the average loop lengths of quadruplexes in the different sets turned out to be very similar.

We next looked further into the individual loop lengths of the three loops (loop 1, 2 and 3 numbered 5' to 3' end) of the G-quadruplex motif. The G-quadruplex motifs with more than four GGG repeats are not considered for this analysis due to the uncertainty regarding which of the actual GGG repeats contribute to the 'stem' of the G-quadruplex motif. However, such sequences represented less than $2 \%$ of all G-quadruplex

Table 2 Quantitative Real Time PCR in HeLa S3 cells after treating with increasing concentrations of different molecules.

\begin{tabular}{|c|c|c|c|c|c|c|c|c|c|}
\hline & CTPS2 & DPY30 & $\mathrm{DIO} 2$ & EGFR & CANX & AK1 & PFKM & SH3GL1 & TGOLN2 \\
\hline 8979A-10 uM & $\begin{array}{l}1.18 \\
(\mathbf{-})\end{array}$ & $\begin{array}{l}-1.23 \\
(\mathbf{-})\end{array}$ & $\begin{array}{l}1.13 \\
(\mathbf{-})\end{array}$ & $\begin{array}{l}-1.79 \\
(\mathbf{-})\end{array}$ & $\begin{array}{l}-1.45 \\
(\mathbf{-})\end{array}$ & $\begin{array}{l}2.02 \\
(\mathbf{m})\end{array}$ & $\begin{array}{l}-1.99 \\
(\mathbf{-})\end{array}$ & $\begin{array}{l}1.61 \\
(\mathbf{-}) \\
\end{array}$ & $\begin{array}{l}-1.29 \\
(\mathbf{-})\end{array}$ \\
\hline 8979A-100 uM & $\begin{array}{l}1.28 \\
(\mathbf{-})\end{array}$ & $\begin{array}{l}1.00 \\
(\mathbf{-})\end{array}$ & $\begin{array}{l}-1.37 \\
(\mathbf{-})\end{array}$ & $\begin{array}{l}1.95 \\
(\mathbf{-})\end{array}$ & $\begin{array}{l}1.14 \\
(\mathbf{-})\end{array}$ & $\begin{array}{l}-1.91 \\
(\mathbf{-})\end{array}$ & $\begin{array}{l}1.06 \\
(\mathbf{-})\end{array}$ & $\begin{array}{l}1.95 \\
(\mathbf{-})\end{array}$ & $\begin{array}{l}1.82 \\
(\mathbf{-})\end{array}$ \\
\hline PhenDC3-10 uM & $\begin{array}{l}3.06 \\
(\wedge)\end{array}$ & $\begin{array}{l}-1.79 \\
(\mathbf{v})\end{array}$ & $\begin{array}{l}1.35 \\
(\mathbf{\Delta})\end{array}$ & $\begin{array}{l}-1.39 \\
(\mathbf{v})\end{array}$ & $\begin{array}{l}12.69 \\
(\mathbf{A})\end{array}$ & $\begin{array}{l}-1.61 \\
(\mathbf{v})\end{array}$ & & & $\begin{array}{l}1.06 \\
(\mathbf{-})\end{array}$ \\
\hline PhenDC3-20 uM & $\begin{array}{l}2.09 \\
(\Delta)\end{array}$ & $\begin{array}{l}-1.83 \\
(\mathbf{v})\end{array}$ & $\begin{array}{l}1.41 \\
(\mathbf{\Delta})\end{array}$ & $\begin{array}{l}-1.65 \\
(\mathbf{v})\end{array}$ & $\begin{array}{l}10.78 \\
(\mathbf{\Delta})\end{array}$ & $\begin{array}{l}-1.41 \\
(\mathbf{v})\end{array}$ & & & \\
\hline PhenDC3-50 uM & $\begin{array}{l}1.93 \\
(\mathbf{\Delta})\end{array}$ & $\begin{array}{l}-3.59 \\
(\mathbf{v})\end{array}$ & $\begin{array}{l}1.99 \\
(\mathbf{\Delta})\end{array}$ & $\begin{array}{l}-1.78 \\
(\mathbf{v})\end{array}$ & $7.06(\mathbf{4})$ & $\begin{array}{l}-1.59 \\
(\mathbf{v})\end{array}$ & & & \\
\hline PhenDC3-100 uM & $\begin{array}{l}1.50 \\
(\mathbf{\Delta})\end{array}$ & $\begin{array}{l}-3.60 \\
(\mathbf{v})\end{array}$ & $\begin{array}{l}2.19 \\
(\mathbf{\Delta})\end{array}$ & $\begin{array}{l}-1.99 \\
(\mathbf{v})\end{array}$ & $\begin{array}{l}6.42 \\
(\Delta)\end{array}$ & $\begin{array}{l}-1.76 \\
(\mathbf{v})\end{array}$ & & & $\begin{array}{l}1.04 \\
(\mathbf{=})\end{array}$ \\
\hline 360A-10 uM & $\begin{array}{l}3.01 \\
(\mathbf{\Delta})\end{array}$ & $\begin{array}{l}-2.06 \\
(\mathbf{v})\end{array}$ & $\begin{array}{l}-1.24 \\
(\mathbf{v})\end{array}$ & $\begin{array}{l}2.41 \\
(\mathbf{4})\end{array}$ & & & $\begin{array}{l}1.86 \\
(\wedge)\end{array}$ & $\begin{array}{l}1.29 \\
(\mathbf{4})\end{array}$ & $\begin{array}{l}1.43 \\
(\mathbf{-})\end{array}$ \\
\hline $360 A-20 u M$ & $\begin{array}{l}1.65 \\
(\mathbf{\Delta})\end{array}$ & $\begin{array}{l}-1.11 \\
(\mathbf{v})\end{array}$ & $\begin{array}{l}-1.08 \\
(\mathbf{v})\end{array}$ & $\begin{array}{l}2.42 \\
(\mathbf{\Delta})\end{array}$ & & & $\begin{array}{l}4.13 \\
(\mathbf{4})\end{array}$ & $\begin{array}{l}3.73 \\
(\mathbf{\Lambda})\end{array}$ & \\
\hline $360 A-50$ uM & $\begin{array}{l}3.73 \\
(\mathbf{\Delta})\end{array}$ & $\begin{array}{l}-1.89 \\
(\mathbf{v})\end{array}$ & $\begin{array}{l}-1.69 \\
(\mathbf{v})\end{array}$ & $\begin{array}{l}6.23 \\
(\Delta)\end{array}$ & & & $\begin{array}{l}1.87 \\
(\mathbf{\Delta})\end{array}$ & $\begin{array}{l}1.25 \\
(\mathbf{\Delta})\end{array}$ & \\
\hline $360 \mathrm{~A}-100 \mathrm{uM}$ & $\begin{array}{l}8.22 \\
(\mathbf{\Delta})\end{array}$ & $\begin{array}{l}-1.22 \\
(\mathbf{v})\end{array}$ & $\begin{array}{l}-2.17 \\
(\mathbf{v})\end{array}$ & $\begin{array}{l}13.11 \\
(\mathbf{\Delta})\end{array}$ & & & $\begin{array}{l}2.23 \\
(\mathbf{\Delta})\end{array}$ & $\begin{array}{l}2.34 \\
(\mathbf{\Delta})\end{array}$ & $\begin{array}{l}-1.12 \\
(\mathbf{- )}\end{array}$ \\
\hline
\end{tabular}

The status in microarray is shown in parenthesis as unchanged $(\boldsymbol{-})$, upregulated $(\mathbf{\Delta})$ or downregulated $(\mathbf{v})$ 


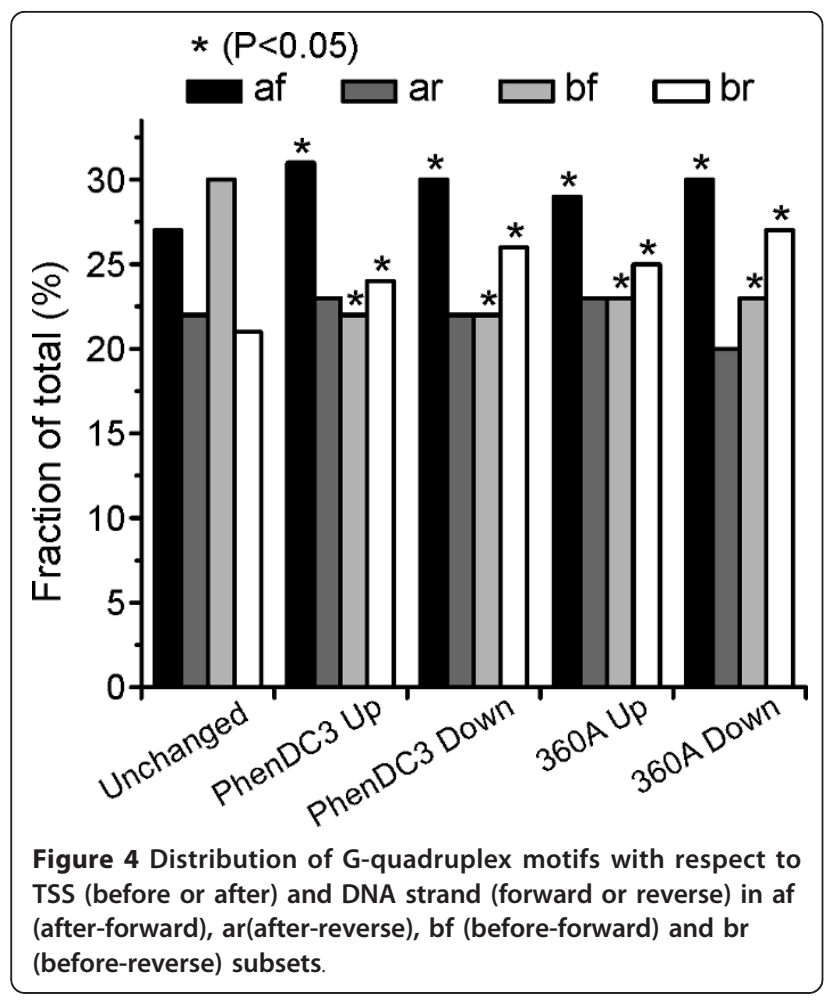

motifs. Interestingly, G-quadruplex motifs in the upregulated genes exhibited significant changes in some loop length combinations (hypergeometric test, PhenDC3: [5-4-3], $\mathrm{P}=3.27 \mathrm{E}^{-06}$ and 360A: [1-3-3], $\mathrm{P}=$ $5.17 \mathrm{E}^{-06} ;[3-7-7], \mathrm{P}=5.52 \mathrm{E}^{-08} ;[6-4-2], \mathrm{P}=6.71 \mathrm{E}^{-07} ;[6-$ $7-2], \mathrm{P}=2.91 \mathrm{E}^{-04} ;[6-7-6], \mathrm{P}=3.39 \mathrm{E}^{-04} ;[7-6-4], \mathrm{P}=$ $3.31 \mathrm{E}^{-08}$; wherein [n-n-n] is [loop1-loop2-loop3], Figure $5 \mathrm{C}$ and $5 \mathrm{E}$, respectively) compared to the mosaic plot of whole genome G-quadruplex loop length compositions and the quadruplexes found in the set of non-differentially expressed genes (Figure 5A and 5B). Although we could find some of the loop combinations significantly enriched using the hypergeometric test, these results could be partially affected due to the low number of Gquadruplexes in the $360 \mathrm{~A}$ up-regulated gene set. Interestingly, the G-quadruplexes belonging to genes with unchanged expression did not show any significant changes in loop composition (Figure 5B). Noticeably, only longer loop length combinations exhibited higher representation in the PhenDC3 and 360A-up-regulated genes. One possible explanation might be that longer loops provide more surface for recognition by interaction partners as also concluded recently [52]. In the presence of G4-binding small molecules these interactions may be hindered leading to differential expression of the corresponding genes. The PhenDC3-down-regulated genes did not show any significant changes (Figure 5D), while the number of G-quadruplexes in 360A-down-

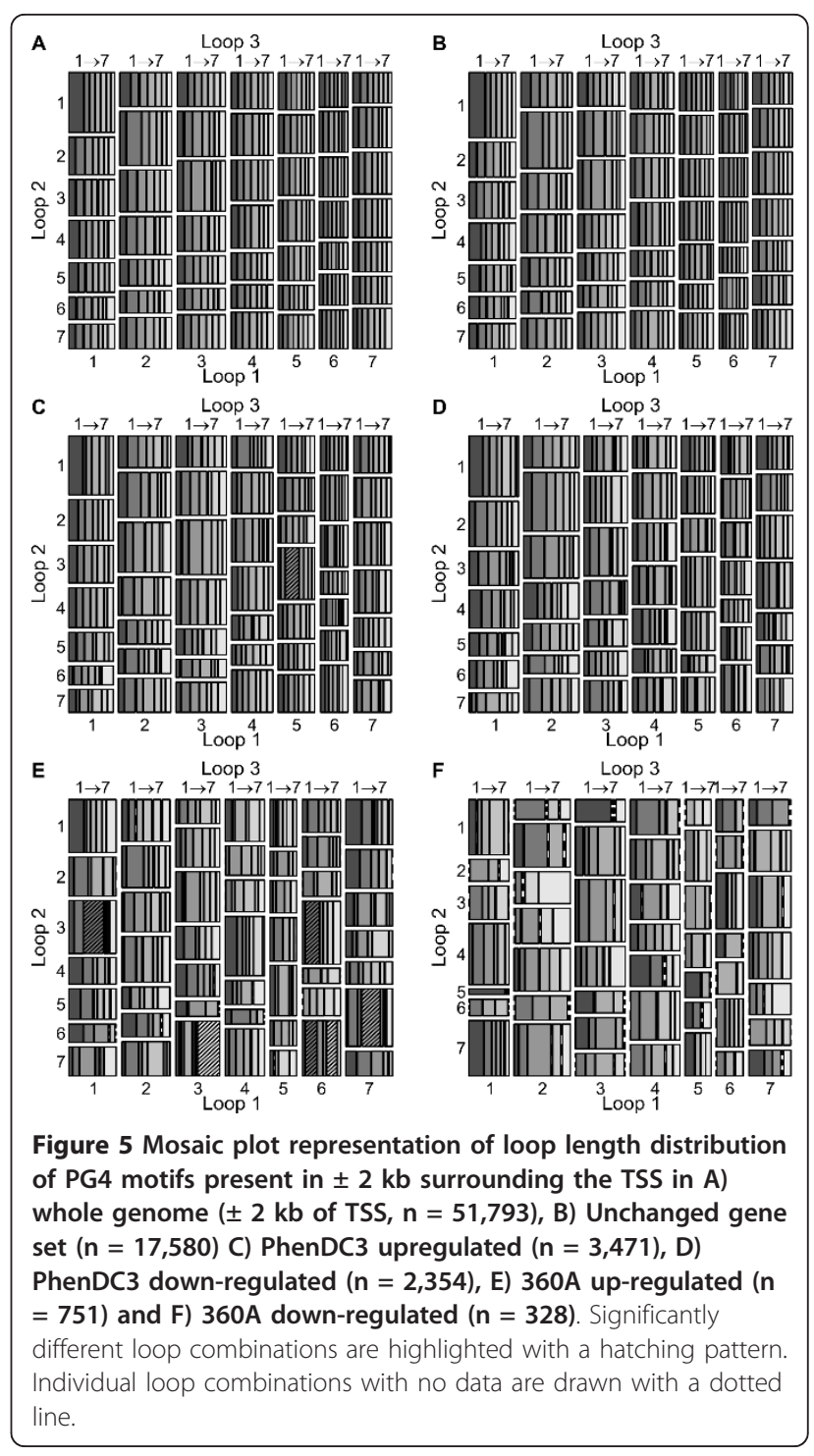

regulated genes $(\mathrm{n}=328)$ are less than the possible loop combinations $(7 \times 7 \times 7=343)$ and hence this set was not tested for significance (Figure 5F).

We further examined the nucleotide composition in the loops of the G-quadruplex motifs in the differentially expressed and the unchanged genes. We checked for the frequency of individual nucleotides (A, C, G, and $\mathrm{T}$ ) as well as groupings (AT, CT and GT) with respect to the total length of the three loops. The grouping AT represents the total number of $\mathrm{A}$ and $\mathrm{T}$ in the loops. The same is true for other groupings. The inverse of these groupings would be CG, AG and AC frequencies, respectively, which, combined, allows us to represent all possible nucleotide combinations. The kernel density plot [53], which is a non-parametric method of estimation of the frequency distribution of a finite and random variable, in present case the nucleotide(s) in the loops, 
were prepared using ' $R$ ' for all the individual nucleotides or groupings in different sets and compared with the reference (non-differential) set (described above). The frequency value can vary from 0 to 1 , where ' 0 ' represents no loop nucleotide matching with the query nucleotide base(s) and ' 1 ' represents that all the nucleotides in the loop region are the same as the query nucleotide base(s). Incidentally, except for nucleotide ' $\mathrm{C}$ ' the frequencies for all other nucleotides or nucleotide groupings in the loop regions were found to have similar distributions (Figure 6). In case of ' $C$ ' occurring in the loop region, $\mathrm{G}$-quadruplexes in the unchanged expression gene set showed a higher incidence of complete exclusion of $C$ (zero frequency) compared to the differentially expressed genes (Kolmogorov-Smirnov test, PhenDC3 up: $\mathrm{P}=1.197 \mathrm{E}^{-09}$, PhenDC3 down: $\mathrm{P}=$ 1.407 $\mathrm{E}^{-04}$, 360A up: $\mathrm{P}=1812 \mathrm{E}^{-05}$, 360A down: $\mathrm{P}=$ 0.497). To further check if the position with respect to TSS or strand has any relevance between the up- and down-regulated genes, we analyzed the kernel density plots for subgroups af, ar, bf, and br (described above) for the differentially expressed genes. However, under these conditions, we could not find any significant changes in the loop distribution of the individual nucleotides or nucleotide groupings (Additional file 1: Figure S1). Taken together, no strong differences of loop compositions were detectable between the sets of genes that showed differential expression after compound treatment and the unchanged genes. However, the binding mode of the utilized bisquinolinium compounds is not known for the many different variations of quadruplexes targeted here. The exact binding mode has probably a large influence on how sequence variations will affect the changes of gene expression observed after compound treatment.

\section{Functional classification of the differentially expressed genes}

To understand the biological relevance of the differentially expressed genes, we performed functional class scoring using Gene Ontology (GO) [54]. The differentially expressed genes after treatment with PhenDC3 exhibited enrichment $(\mathrm{P}<0.01$, after Bonferroni correction) in gene classes involved in various metabolic processes $\left(P=1.19 \mathrm{E}^{-10}\right)$, gene expression $\left(\mathrm{P}=2.15 \mathrm{E}^{-07}\right)$, and regulation of transcription by RNA pol II ( $\mathrm{P}=$ $0.0003)$. Similar enrichment of metabolic pathways (transcription regulation by RNA pol II) was noticed earlier [53]. On the other hand, genes belonging to the immune response $(P=0.0002)$ and $G$-protein-coupled signal transduction $(\mathrm{P}=0.006)$ were significantly depleted (Table 3 ). The first result is likely caused by the fact that most genes in this class are silent in the examined cell line. In case of $360 \mathrm{~A}$ treated cells no GO
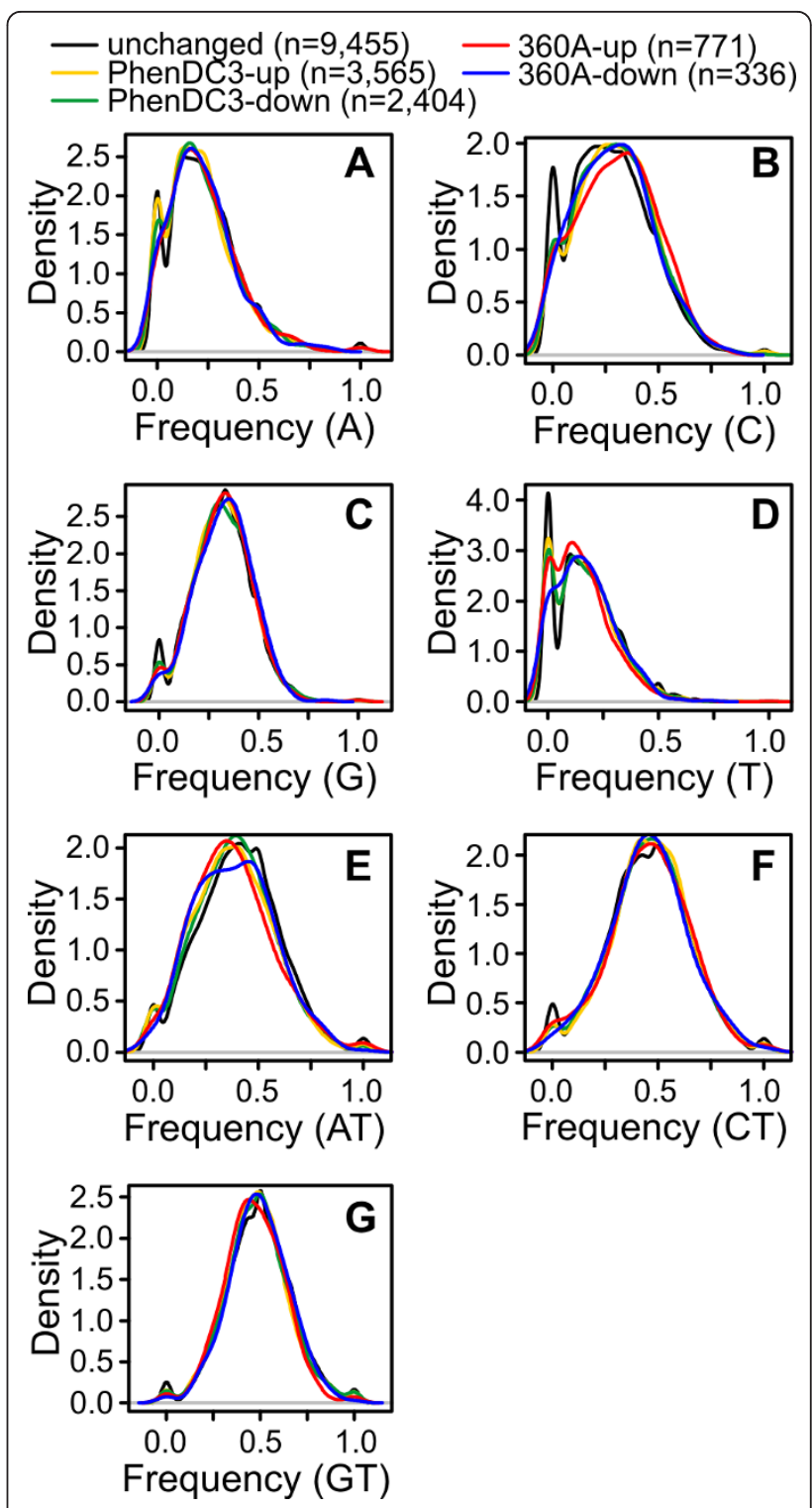

Figure 6 Loop composition analysis of G-quadruplex motifs present in differentially expressed genes. Kernel density plot showing the distribution of individual nucleotides (A) A, (B) C, (C) G and (D) T or nucleotide groupings (E) AT, (F) CT and (G) GT that

occur in loops of G-quadruplex motifs. The number of G-quadruplex motifs used to plot the kernel density is shown in the parentheses.

class was significantly enriched or depleted after correction for multiple testing, however, without Bonferroni correction, enrichment in classes for cell cycle, protein transport, and localization was found (Additional file 1: Table S3) which was also shown for TMPyP4-treated cells before [55]. The enrichment of the differentially expressed genes in various metabolic processes and protein transport pathways could pose a challenge for estimating the therapeutic consequences of G-quadruplex specific molecules $[19,56]$ which are thought to act at 
Table 3 Gene Ontology (GO) categories enriched/depleted $(P<0.01)$ of differentially expressed genes after treating HeLa S3 cells with PhenDC3

\begin{tabular}{|c|c|c|}
\hline GO ID & TERM & P VALUE \\
\hline \multicolumn{3}{|c|}{ Enriched GO classes } \\
\hline GO:0044237 & cellular metabolic process & $2.42 \mathrm{E}-12$ \\
\hline GO:0034960 & cellular biopolymer metabolic process & 5.07E-12 \\
\hline GO:0044260 & cellular macromolecule metabolic process & $2.90 \mathrm{E}-11$ \\
\hline GO:0043283 & biopolymer metabolic process & $5.91 \mathrm{E}-11$ \\
\hline GO:0008152 & metabolic process & $1.19 \mathrm{E}-10$ \\
\hline GO:0043170 & macromolecule metabolic process & $2.94 \mathrm{E}-10$ \\
\hline GO:0044238 & primary metabolic process & $8.10 \mathrm{E}-10$ \\
\hline GO:0006139 & nucleobase, nucleoside, nucleotide and nucleic acid metabolic process & $1.91 \mathrm{E}-07$ \\
\hline GO:0010467 & gene expression & $2.15 \mathrm{E}-07$ \\
\hline GO:0009987 & cellular process & 4.16E-07 \\
\hline GO:0043284 & biopolymer biosynthetic process & $1.28 \mathrm{E}-06$ \\
\hline GO:0034961 & cellular biopolymer biosynthetic process & $1.28 \mathrm{E}-06$ \\
\hline GO:0016070 & RNA metabolic process & $1.90 \mathrm{E}-06$ \\
\hline GO:0034645 & cellular macromolecule biosynthetic process & 5.34E-06 \\
\hline GO:0009059 & macromolecule biosynthetic process & $8.73 \mathrm{E}-06$ \\
\hline GO:0046907 & intracellular transport & 0.0001430 \\
\hline GO:0051641 & cellular localization & 0.0002268 \\
\hline GO:0006357 & regulation of transcription from RNA polymerase II promoter & 0.0003508 \\
\hline GO:0044249 & cellular biosynthetic process & 0.0007565 \\
\hline GO:0009058 & biosynthetic process & 0.0009248 \\
\hline GO:0051649 & establishment of localization in cell & 0.0013971 \\
\hline GO:0044267 & cellular protein metabolic process & 0.0075149 \\
\hline \multicolumn{3}{|c|}{ Depleted GO classes } \\
\hline GO:0002376 & immune system process & 0.0074009 \\
\hline GO:0007186 & G-protein coupled receptor protein signaling pathway & 0.0063202 \\
\hline GO:0007166 & cell surface receptor linked signal transduction & 0.0002492 \\
\hline GO:0006955 & immune response & 0.0001782 \\
\hline GO:0032501 & multicellular organismal process & 4.93E-06 \\
\hline
\end{tabular}

least in part via suppression of transcription of individual oncogenes $[20,25,28,57]$.

\section{Conclusions}

In summary, the present study shows that the bisquinolinium compounds PhenDC3 and 360A can induce Gquadruplex-motif-specific changes in transcription. In contrast to previous studies, our experiments employ more quadruplex-specific compounds, which is reflected in the over-representation of differentially regulated genes bearing quadruplex motifs in their promoter regions. In an earlier study using TMPyP4 as a more non-specific Gquadruplex binder $61 \%$ of the differentially expressed genes were connected to the occurrence of a G4 motif in their $\pm 1 \mathrm{~kb}$ region around TSS which is less than expected [5]. In comparison, PhenDC3 and 360A showed enhanced specificity. The differences observed between the two compounds can result from slight variations in affinity for various quadruplex-forming sequences as shown recently [58]. However, we were unable to detect obvious characteristics such as loop compositions and motifs common to quadruplexes that are associated with genes that showed changes. Such identifiers would also have been very interesting to observe also with respect to the therapeutic potential of quadruplex-targeting small molecules. On the other hand, given the rather late time point of $48 \mathrm{~h}$ post incubation with the quadruplex-inducing compound, we cannot rule out that some or many of the observed effects are secondary and therefore cannot be attributed to a direct effect of a quadruplex formation. Taking this into account, future studies of quadruplex-targeting compounds should also take into account earlier time points in order to ensure that indeed primary changes of gene expression are observed. 
Interestingly, we found that more genes were up-regulated than down-regulated following compound treatment. Several studies reported quadruplexes as transcription suppressor elements when their effects on individual genes were studied [19,28,59]. However, positive effects on transcription levels via the formation of promoter G-quadruplexes have also been described $[60,61]$ and hence the influence of the formation of a promoter-based quadruplex on transcription is very likely dependent on the exact position and the promoter architecture. Similar up-regulation effects were noticed earlier in genome-wide microarray experiments following TMPyP4 [55] or single-chain antibody [38] treatments. Similarly, in absence of WRN/BLM helicases which are believed to resolve G-quadruplex motifs, a strong association was observed between up-regulated genes and G-quadruplex motifs in promoter regions [62]. It has been speculated that the changes could be mediated by an influence of the G4 motifs on the chromatin state $[10,12]$. It is also possible that the absence of a helicase (or in our case stabilization of transient Gquadruplex motifs by small molecules) may result in formation of transcription factor binding sites or destruction of repressor binding sites. In addition, along with other non-canonical structures such as cruciform and triplexes, quadruplexes have been discussed as modulators of superhelical torsion. Stress-induced duplex destabilization (SIDD) is more prevalent in transcriptional regulatory sites [63] and quadruplex formation was shown to release supercoiling stress [6]. The occurring denaturation of duplex DNA in regulatory regions near the transcription start site would be an easy way of interfering with the transcriptional machinery and its regulation. However, more detailed studies are required to further decipher the exact mechanisms underlying quadruplex-mediated changes in transcription. We also observed a positional bias in the distribution of G-quadruplex motifs in differentially expressed genes. G-quadruplexes are enriched on the reverse strand before the TSS or on the forward strand after the TSS when compared to the G4-motifs present in non-differentially expressed genes. Interestingly, G4 motifs present on the non-template strand have been shown to play a role in RNA pol II pausing [64], which provides a possible mechanism for how these may in turn regulate the expression of the downstream gene. Further, a similar distribution of the loop size and composition of G-quadruplexes between the perturbed and non-perturbed genes were found, suggesting the precise genomic position and the composition of the surrounding DNA are likely to play an important role in determining the effect a given quadruplex will have on gene-transcription.

\section{Methods}

\section{RNA Isolation and microarray experiments}

HeLa S3 cells (obtained from the DSMZ, the German Resource Center for Biological Materials, http://www. dsmz.de/) were cultured in DMEM high glucose medium with 10\% Fetal Calf Serum (FCS). For PhenDC3, 360A and 8979A, $10 \mathrm{mM}$ stock solutions were prepared in DMSO and diluted directly in the DMEM medium to $10 \mu \mathrm{M}$ just before using. The HeLa S3 cells exhibited $92 \%$ and $100 \%$ cell viability at $10 \mu \mathrm{M}$ PhenDC3 and $360 \mathrm{~A}$, respectively, after $48 \mathrm{hrs}$ treatment. After 48 hours of treatment, the whole RNA was isolated using RNeasy plus mini kit (Qiagen). Microarray experiment was performed by Aros Applied Biotechnology (Denmark) as per the manufacturer's protocol. Briefly, RNA was amplified using Illumina TotalPrep RNA amplification kit and hybridized on Illumina HumanHT-12v4 expression BeadChips as recommended by the manufacturer. Hybridization data was obtained by iScan Bead Array scanner (Illumina) and preprocessed by quantile normalization (Compliance to MIAME guidelines).

\section{Microarray data analysis}

The intensity values after quantile normalization for all triplicate experiments were further analyzed using CLANS software http://bioinfoserver.rsbs.anu.edu.au/ downloads/clans/. Briefly, all the Illumina IDs (probes) were assumed to be differentially expressed if their anova values were $\leq 0.5$ and to be of interest if their correlation to a condition of interest (see above, table or figure listing the 8 conditions) was $\geq 0.9$. All experiments were performed in triplicate and overall > 98\% correlation was observed between replicates.

\section{Data retrieval and G-quadruplex mapping}

The Genbank (.gbk)files for the Homo sapiens reference genome build 36.2 were downloaded from the NCBI ftp site ftp://ftp.ncbi.nih.gov/genomes/H_sapiens/ARCHIVE/ BUILD.36.2. The G-quadruplex motifs were identified using a custom PERL program. The motif searched for was 5' $-\left(G_{G G N}{ }_{1-7}\right)_{\geq 3}$ GGG-3', wherein $\mathrm{N}=\mathrm{A}$, T, G or C. All G-quadruplex motifs were mapped to their nearest mRNA within $\pm 2 \mathrm{~kb}$ of Transcription Start Sites (TSS). To be certain the same sequence build was used for all analyses, we independently mapped the Illumina probe sequences $(47,323)$ onto the available mRNA data using BLAT [65] and also the mRNA sequences $(45,495$; retrieved from the Genbank files) onto the Illumina IDs. One Illumina ID may map to multiple mRNAs and vice versa, due to the non-equivalence of the datasets. This resulted in 32,980 mRNAs mapping to 34,638 Illumina probe IDs. The 32,980 mRNAs could be matched to 24,651 NCBI genes. Next, we also mapped the G- 
quadruplexes present $(53,397)$ in the $\pm 2 \mathrm{~kb}$ region of the TSS of the 17,384 mRNAs (16,607 genes). The terms "forward", "reverse", "before TSS", and "after TSS" used to classify quadruplexes refer to the position the G-rich quadruplex is found with respect to the coding (sense or template) strand of the associated gene. In this respect "forward" means the presence of a quadruplex on the coding (sense or template) strand.

\section{Real time polymerase chain reaction}

Two step real time PCR was performed to validate the microarray results. cDNA was prepared by using $\mathrm{M}$ MuLV reverse transcriptase (Fermentas). Relative quantitative real time PCR was performed using LightCycler 480 SYBR Green I master (Roche) in a Roche LightCycler 480 system, for primer sequences see additional file 1, Figure S2. A negative control for each gene with no template was included. Reaction conditions consisted of $95^{\circ} \mathrm{C}$ for 1 min followed by 45 cycles of amplification $\left(15 \mathrm{sec}\right.$ at $95^{\circ} \mathrm{C}, 15 \mathrm{sec}$ at $60^{\circ} \mathrm{C}$ and $15 \mathrm{sec}$ at $72^{\circ} \mathrm{C}$ ). After amplification, melting analysis was performed to ensure a uniform amplification product.

\section{Loop length and composition analysis}

The different features (length and composition) of the G-quadruplex motifs were extracted via a PERL script and visualized using ' $R$ ' http://www.r-project.org/. For programmatic simplicity, the first three guanines from 5 '-end were considered part of the stem and consecutive guanines, if any, were considered as part of the loop (length 1-7). To reduce redundancy in loop length and composition, each G-quadruplex motif was considered only once. All the statistical tests were performed in ' $R$ ' http://www.r-project.org/.

\section{Gene Ontology (GO) classification}

For functional classification all the differentially expressed gene names were converted to Uniprot standard gene name using gene merge (http://genemerge. cbcb.umd.edu/GeneMerge.php, [66]) and these converted gene names were used to obtain the GO category for biological function (http://genome.crg.es/GOToolBox/, [54]). Bonferroni correction was used to correct for multiple testing for PhenDC3. NOTE: The results for the 360A treated sample were not corrected for multiple testing as this removed all significant hits. These results should be interpreted with this in mind. Categories having $\mathrm{p}$-value less than 0.01 was considered to be significantly enriched/depleted.

\section{Availability of supplementary data}

Further data sets including are available as Additional file 1 (containing Figure S1 and Tables S1, S2, and S3) and Additional File 2 (Table S4). The microarray data set supporting the results of this article is available online at the Gene Expression Omnibus repository (accession number GSE32170, http://www.ncbi.nlm.nih. gov/geo/query/acc.cgi?token=rnaxlqiuomkuips\&acc $=$ GSE 3217)

\section{Additional material}

Additional file 1: Table S1. Number of G-quadruplexes present in different subsets. Table S2. Primers used for quantitative real time PCR. Table S3. Enriched Gene Ontology (GO) classes of differentially expressed genes after treating HeLa S3 cells with 360A. Figure S1. Kernel density plot showing the distribution of individual nucleotides (A) A, (B) C, (C) G and (D) T or nucleotide groupings (E) AT, (F) CT and (G) GT that occur in loops of G-quadruplex motifs in af, ar, bf and br subsets (see Table S2).

Additional file 2: Table S4 in excel-format: List of all differentially expressed genes.

\section{Acknowledgements}

We thank Kangkan Halder for helpful discussions. The work was funded by the VolkswagenStiftung via a Lichtenberg Professorship to JSH. JFR received funds from the "Ligue Nationale Contre le Cancer". MPTF and JFR thank the Agence Nationale pour la Recherche ANR (G4-TOOLBOX, ANR-Blan-09-355) for fundings. The funders had no role in study design, data collection and analysis, decision to publish, or preparation of the manuscript.

\section{Author details}

'Department of Chemistry and Konstanz Research School Chemical Biology (KoRS-CB), University of Konstanz, Universitätsstraße 10, 78457 Konstanz, Germany. ${ }^{2}$ Structure des Acides Nucléiques, Télomères et Evolution, INSERM U565, CNRS UMR 7196, Muséum National d'Histoire Naturelle, 43 rue Cuvier 75231 Paris cedex 05, France. ${ }^{3}$ Institut Curie, UMR 176-CNRS, Bât 110 , Université Paris-Sud, 91405 Orsay, France. ${ }^{4}$ Department of Biology, University of Konstanz, Universitätsstraße 10, 78457 Konstanz, Germany.

\section{Authors' contributions}

Conceived and designed the experiments: All authors. Performed the experiments: $\mathrm{RH}$. Analyzed the data: $\mathrm{RH}, \mathrm{TF}, \mathrm{JSH}$. Contributed reagents: $\mathrm{RH}$ JFR MPTF TF. Wrote the manuscript: RH, TF, JSH. All authors read and approved the final manuscript

\section{Competing interests}

The authors declare that they have no competing interests.

Received: 15 November 2011 Accepted: 13 March 2012

Published: 13 March 2012

\section{References}

1. Burge S, Parkinson GN, Hazel P, Todd AK, Neidle S: Quadruplex DNA: sequence, topology and structure. Nucleic Acids Res 2006, 34:5402-5415.

2. Todd AK, Johnston M, Neidle S: Highly prevalent putative quadruplex sequence motifs in human DNA. Nucleic Acids Res 2005, 33:2901-2907.

3. Huppert $\lrcorner \mathrm{L}$, Balasubramanian $\mathrm{S}$ : Prevalence of quadruplexes in the human genome. Nucleic Acids Res 2005, 33:2908-2916.

4. Du Z, Zhao Y, Li N: Genome-wide analysis reveals regulatory role of G4 DNA in gene transcription. Genome Res 2008, 18:233-241.

5. Verma A, Halder K, Halder R, Yadav VK, Rawal P, Thakur RK, Mohd F, Sharma A, Chowdhury S: Genome-wide computational and expression analyses reveal G-quadruplex DNA motifs as conserved cis-regulatory elements in human and related species. J Med Chem 2008, 51:5641-5649.

6. Rawal P, Kummarasetti VB, Ravindran J, Kumar N, Halder K, Sharma R, Mukerji M, Das SK, Chowdhury S: Genome-wide prediction of G4 DNA as regulatory motifs: role in Escherichia coli global regulation. Genome Res 2006, 16:644-655. 
7. Kumari S, Bugaut A, Huppert JL, Balasubramanian S: An RNA G-quadruplex in the $5^{\prime}$ UTR of the NRAS proto-oncogene modulates translation. Nat Chem Biol 2007, 3:218-221.

8. Halder K, Wieland $M$, Hartig JS: Predictable suppression of gene expression by 5'-UTR-based RNA quadruplexes. Nucleic Acids Res 2009, 37:6811-6817.

9. Paeschke K, Capra JA, Zakian VA: DNA replication through G-quadruplex motifs is promoted by the Saccharomyces cerevisiae Pif1 DNA helicase. Cell 2011, 145:678-691.

10. Halder K, Halder R, Chowdhury S: Genome-wide analysis predicts DNA structural motifs as nucleosome exclusion signals. Mol Biosyst 2009, 5:1703-1712

11. Wong HM, Huppert JL: Stable G-quadruplexes are found outside nucleosome-bound regions. Mol Biosyst 2009, 5:1713-1719.

12. Halder R, Halder K, Sharma P, Garg G, Sengupta S, Chowdhury S: Guanine quadruplex DNA structure restricts methylation of $\mathrm{CpG}$ dinucleotides genome-wide. Mol Biosyst 2010, 6:2439-2447.

13. Mani P, Yadav VK, Das SK, Chowdhury S: Genome-wide analyses of recombination prone regions predict role of DNA structural motif in recombination. PLoS One 2009, 4:e4399.

14. Kostadinov R, Malhotra N, Viotti M, Shine R, D'Antonio L, Bagga P: GRSDB: a database of quadruplex forming G-rich sequences in alternatively processed mammalian pre-mRNA sequences. Nucleic Acids Res 2006, 34 D119-D124.

15. Huppert JL, Balasubramanian S: G-quadruplexes in promoters throughout the human genome. Nucleic Acids Res 2007, 35:406-413.

16. Qin Y, Hurley LH: Structures, folding patterns, and functions of intramolecular DNA G-quadruplexes found in eukaryotic promoter regions. Biochimie 2008, 90:1149-1171.

17. Huppert JL: Structure, location and interactions of G-quadruplexes. FEBS 」 2010, 277:3452-3458

18. Brooks TA, Kendrick S, Hurley L: Making sense of G-quadruplex and imotif functions in oncogene promoters. FEBS J 2010, 277:3459-3469.

19. Balasubramanian S, Hurley LH, Neidle S: Targeting G-quadruplexes in gene promoters: a novel anticancer strategy? Nat Rev Drug Discov 2011 10:261-275.

20. Siddiqui-Jain A, Grand CL, Bearss DJ, Hurley LH: Direct evidence for a Gquadruplex in a promoter region and its targeting with a small molecule to repress c-MYC transcription. Proc Natl Acad Sci USA 2002, 99:11593-11598.

21. Brown RV, Danford FL, Gokhale V, Hurley LH, Brooks TA: Demonstration that Drug-targeted Down-regulation of MYC in Non-Hodgkins Lymphoma Is Directly Mediated through the Promoter G-quadruplex. J Biol Chem 2011, 286:41018-41027.

22. Palumbo SL, Memmott RM, Uribe DJ, Krotova-Khan Y, Hurley LH, Ebbinghaus SW: A novel G-quadruplex-forming GGA repeat region in the c-myb promoter is a critical regulator of promoter activity. Nucleic Acids Res 2008, 36:1755-1769.

23. Sun D, Liu W-J, Guo K, Rusche JJ, Ebbinghaus S, Gokhale V, Hurley LH: The proximal promoter region of the human vascular endothelial growth factor gene has a G-quadruplex structure that can be targeted by Gquadruplex-interactive agents. Mol Cancer Ther 2008, 7:880-889.

24. McLuckie K, Waller Z, Sanders D, Alves D, Rodriguez R, Dash J, McKenzie G, Venkitaraman A, Balasubramanian S: G-quadruplex-binding benzo[a] phenoxazines down-regulate c-KIT expression in human gastric carcinoma cells. J Am Chem Soc 2011, 133:2658-2663.

25. Cogoi S, Xodo LE: G-quadruplex formation within the promoter of the KRAS proto-oncogene and its effect on transcription. Nucleic Acids Res 2006, 34:2536-2549.

26. Membrino A, Cogoi S, Pedersen E, Xodo L: G4-DNA formation in the HRAS promoter and rational design of decoy oligonucleotides for cancer therapy. PLoS One 2011, 6:e24421.

27. Qin Y, Fortin J, Tye D, Gleason-Guzman M, Brooks T, Hurley L: Molecular cloning of the human platelet-derived growth factor receptor beta (PDGFR-beta) promoter and drug targeting of the G-quadruplex-forming region to repress PDGFR-beta expression. Biochemistry 2010, 49.4208-4219.

28. Wang XD, Ou TM, Lu YJ, Li Z, Xu Z, Xi C, Tan JH, Huang SL, An LK, Li D, et al: Turning off transcription of the bcl-2 gene by stabilizing the bcl-2 promoter quadruplex with quindoline derivatives. J Med Chem 2010, 53:4390-4398.
29. Patel DJ, Phan AT, Kuryavyi V: Human telomere, oncogenic promoter and 5'-UTR G-quadruplexes: diverse higher order DNA and RNA targets for cancer therapeutics. Nucleic Acids Res 2007, 35:7429-7455.

30. Wong HM, Payet L, Huppert JL: Function and targeting of Gquadruplexes. Curr Opin Mol Ther 2009, 11:146-155.

31. Anantha NV, Azam M, Sheardy RD: Porphyrin binding to quadrupled T4G4. Biochemistry 1998, 37:2709-2714.

32. Mikami-Terao Y, Akiyama M, Yuza Y, Yanagisawa T, Yamada O, Yamada H: Antitumor activity of G-quadruplex-interactive agent TMPyP4 in K562 leukemic cells. Cancer Lett 2008, 261:226-234.

33. De Cian A, Guittat L, Shin-ya K, Riou JF, Mergny JL: Affinity and selectivity of G4 ligands measured by FRET. Nucleic Acids Symp Ser (Oxf) 2005, 49:235-236.

34. Liu W, Sun D, Hurley LH: Binding of G-quadruplex-interactive agents to distinct G-quadruplexes induces different biological effects in MiaPaCa cells. Nucleosides Nucleotides Nucleic Acids 2005, 24:1801-1815.

35. Izbicka E, Wheelhouse RT, Raymond E, Davidson KK, Lawrence RA, Sun D, Windle BE, Hurley LH, Von Hoff DD: Effects of cationic porphyrins as Gquadruplex interactive agents in human tumor cells. Cancer Res 1999, 59:639-644.

36. Snyder JW, Lambert JD, Ogillby PR: 5,10,15,20-tetrakis(N-methyl-4-pyridyl)$21 \mathrm{H}, 23 \mathrm{H}$-porphine (TMPyP) as a sensitizer for singlet oxygen imaging in cells: characterizing the irradiation-dependent behavior of TMPyP in a single cell. Photochem Photobiol 2006, 82:177-184.

37. Tada-Oikawa S, Oikawa S, Hirayama J, Hirakawa K, Kawanishi S: DNA damage and apoptosis induced by photosensitization of 5,10,15,20 tetrakis (N-methyl-4-pyridyl)-21H,23H-porphyrin via singlet oxygen generation. Photochem Photobiol 2009, 85:1391-1399,

38. Fernando H, Sewitz S, Darot J, Tavare S, Huppert JL, Balasubramanian S: Genome-wide analysis of a G-quadruplex-specific single-chain antibody that regulates gene expression. Nucleic Acids Res 2009, 37:6716-6722.

39. Muller S, Kumari S, Rodriguez R, Balasubramanian S: Small-moleculemediated G-quadruplex isolation from human cells. Nat Chem 2010 2:1095-1098.

40. Riou JF, Guittat L, Mailliet P, Laoui A, Renou E, Petitgenet O, MegninChanet $F$, Helene $C$, Mergny JL: Cell senescence and telomere shortening induced by a new series of specific G-quadruplex DNA ligands. Proc Natl Acad Sci USA 2002, 99:2672-2677.

41. Monchaud D, Allain C, Bertrand H, Smargiasso N, Rosu F, Gabelica V, De Cian A, Mergny JL, Teulade-Fichou MP: Ligands playing musical chairs with G-quadruplex DNA: a rapid and simple displacement assay for identifying selective G-quadruplex binders. Biochimie 2008, 90:1207-1223.

42. De Cian A, Cristofari G, Reichenbach P, De Lemos E, Monchaud D, TeuladeFichou MP, Shin-Ya K, Lacroix L, Lingner J, Mergny JL: Reevaluation of telomerase inhibition by quadruplex ligands and their mechanisms of action. Proc Natl Acad Sci USA 2007, 104:17347-17352.

43. Pennarun G, Granotier C, Gauthier LR, Gomez D, Hoffschir F, Mandine E, Riou JF, Mergny JL, Mailliet P, Boussin FD: Apoptosis related to telomere instability and cell cycle alterations in human glioma cells treated by new highly selective G-quadruplex ligands. Oncogene 2005, 24:2917-2928.

44. Pennarun G, Granotier C, Hoffschir F, Mandine E, Biard D, Gauthier LR, Boussin FD: Role of ATM in the telomere response to the G-quadruplex ligand 360A. Nucleic Acids Res 2008, 36:1741-1754.

45. Granotier C, Pennarun G, Riou L, Hoffschir F, Gauthier LR, De Cian A, Gomez D, Mandine E, Riou JF, Mergny JL, et al: Preferential binding of a G-quadruplex ligand to human chromosome ends. Nucleic Acids Res 2005, 33:4182-4190.

46. Piazza A, Boule JB, Lopes J, Mingo K, Largy E, Teulade-Fichou MP, Nicolas A: Genetic instability triggered by G-quadruplex interacting Phen-DC compounds in Saccharomyces cerevisiae. Nucleic Acids Res 2010, 38:4337-4348.

47. Halder K, Largy E, Benzler M, Teulade-Fichou MP, Hartig JS: Efficient suppression of gene expression by targeting 5'-UTR-based RNA quadruplexes with bisquinolinium compounds. Chembiochem 2011, 12:1663-1668

48. Frickey T, Weiller G: Analyzing microarray data using CLANS. Bioinformatics 2007, 23:1170-1171.

49. Guedin A, Gros J, Alberti P, Mergny JL: How long is too long? Effects of loop size on G-quadruplex stability. Nucleic Acids Res 2010, 38:7858-7868. 
50. Jaumot J, Gargallo R: Using principal component analysis to find correlations between loop-related and thermodynamic variables for Gquadruplex-forming sequences. Biochimie 2010, 92:1016-1023.

51. Hazel P, Huppert J, Balasubramanian S, Neidle S: Loop-length-dependent folding of G-quadruplexes. J Am Chem Soc 2004, 126:16405-16415.

52. Takahama K, Sugimoto C, Arai S, Kurokawa R, Oyoshi T: Loop lengths of Gquadruplex structures affect the G-quadruplex DNA binding selectivity of the RGG motif in Ewing's sarcoma. Biochemistry 2011, 50:5369-5378.

53. Shu G, Zeng B, Chen YP, Smith OH: Performance assessment of kernel density clustering for gene expression profile data. Comp Funct Genomics 2003, 4:287-299

54. Martin D, Brun C, Remy E, Mouren P, Thieffry D, Jacq B: GOToolBox: functional analysis of gene datasets based on Gene Ontology. Genome Biol 2004, 5:R101.

55. Verma A, Yadav VK, Basundra R, Kumar A, Chowdhury S: Evidence of genome-wide G4 DNA-mediated gene expression in human cancer cells. Nucleic Acids Res 2009, 37:4194-4204.

56. Balasubramanian S, Neidle S: G-quadruplex nucleic acids as therapeutic targets. Curr Opin Chem Biol 2009, 13:345-353.

57. McLuckie Kl, Waller ZA, Sanders DA, Alves D, Rodriguez R, Dash J, McKenzie GJ, Venkitaraman AR, Balasubramanian S: G-quadruplex-binding benzo[a]phenoxazines down-regulate c-KIT expression in human gastric carcinoma cells. J Am Chem Soc 2011, 133:2658-2663.

58. Tran P, Largy E, Hamon F, Teulade-Fichou M, Mergny J: Fluorescence intercalator displacement assay for screening G4 ligands towards a variety of G-quadruplex structures. Biochimie 2011, 93:1288-1296.

59. Eddy J, Maizels N: Selection for the G4 DNA motif at the $5^{\prime}$ end of human genes. Mol Carcinog 2009, 48:319-325.

60. Cogoi S, Paramasivam M, Membrino A, Yokoyama KK, Xodo LE: The KRAS Promoter Responds to Myc-associated Zinc Finger and Poly(ADP-ribose) Polymerase 1 Proteins, Which Recognize a Critical Quadruplex-forming GA-element. J Biol Chem 2010, 285:22003-22016.

61. Shklover J, Weisman-Shomer P, Yafe A, Fry M: Quadruplex structures of muscle gene promoter sequences enhance in vivo MyoD-dependent gene expression. Nucleic Acids Res 2010, 38:2369-2377.

62. Johnson JE, Cao K, Ryvkin P, Wang LS, Johnson FB: Altered gene expression in the Werner and Bloom syndromes is associated with sequences having G-quadruplex forming potential. Nucleic Acids Res 2010, 38:1114-1122.

63. Benham CJ: Duplex destabilization in superhelical DNA is predicted to occur at specific transcriptional regulatory regions. J Mol Biol 1996, 255:425-434.

64. Eddy J, Vallur AC, Varma S, Liu H, Reinhold WC, Pommier Y, Maizels N: G4 motifs correlate with promoter-proximal transcriptional pausing in human genes. Nucleic Acids Res 2011, 39:4975-4983.

65. Kent WJ: BLAT-the BLAST-like alignment tool. Genome Res 2002, 12:656-664.

66. Castillo-Davis Cl, Hartl DL: GeneMerge-post-genomic analysis, data mining, and hypothesis testing. Bioinformatics 2003, 19:891-892.

doi:10.1186/1756-0500-5-138

Cite this article as: Halder et al.: Bisquinolinium compounds induce quadruplex-specific transcriptome changes in HeLa S3 cell lines. BMC Research Notes 2012 5:138.

\section{Submit your next manuscript to BioMed Central and take full advantage of:}

- Convenient online submission

- Thorough peer review

- No space constraints or color figure charges

- Immediate publication on acceptance

- Inclusion in PubMed, CAS, Scopus and Google Scholar

- Research which is freely available for redistribution

Submit your manuscript at www.biomedcentral.com/submit
Ciomed Central 